\title{
IMPACTO ECONÓMICO DEL CAMBIO CLIMÁTICO SOBRE LA AGRICULTURA EN REGADÍO DE LA DEMARCACIÓN HIDROGRÁFICA DEL SEGURA
}

\author{
$\underline{\text { Francisco Pellicer Martínez }}^{a *}$, Ángel Perni $^{b}$ y José Miguel Martínez Paz ${ }^{c}$ \\ ${ }^{a}$ Departamento de Ingeniería Civil, Universidad Católica de Murcia (Murcia, fpellicer@ucam.edu) \\ ${ }^{b}$ Departamento de Economía Aplicada, Universidad de Cádiz (Cádiz, angel.perni@uca.es) \\ ${ }^{c}$ Departamento de Economía Aplicada, Universidad de Murcia (Murcia, jmpaz@um.es)
}

\section{Resumen}

El objetivo de este trabajo es cuantificar el impacto económico que el Cambio Climático (CC) tendrá sobre la agricultura en regadío de la Demarcación Hidrográfica del Segura (DHS) y valorar posibles medidas de adaptación al mismo. Esta cuenca mediterránea semiárida cuenta con un complejo sistema de gestión de recursos hídricos, pudiendo servir como ejemplo de adaptación a otras cuencas de similares características. Para ello, se simulan tres escenarios climáticos, uno actual que sirve de marco de referencia y otros dos para las proyecciones climáticas futuras (RCP 4.5 y RCP 8.5). La metodología se resume en tres fases secuenciales: evaluación de recursos hídricos, modelización integral del sistema de explotación de la cuenca y modelización de los principales sistemas de regadío de la DHS. En términos agregados, se predice una reducción de la superficie de regadío del -4,7\% en el escenario RCP 4.5 y del -7,4\% en el escenario RCP 8.5, con un impacto económico medio en términos de pérdidas de margen bruto, del $-4,0 \%$ y del $-7,3 \%$, respectivamente.

Palabras clave: Simulación numérica, Gestión de Recursos Hídricos, Planes de cultivo, Riesgo, Sequía.

\section{Introducción y objetivos}

El Cambio Climático (CC) es una de las mayores amenazas para la disponibilidad de recursos hídricos en régimen natural en el área mediterránea, dado que la mayoría de las proyecciones climáticas pronostican una muy importante reducción de los mismos. El sector agrícola será uno de los más afectados, ya que tiene menor prioridad de acceso al recurso agua frente a otros sectores, como el abastecimiento o la industria. Un ejemplo singular en la zona es la Demarcación Hidrográfica del Segura (DHS), que posee un complejo sistema de gestión de recursos hídricos formado por una densa red de infraestructuras hidráulicas con acceso a diferentes fuentes de recurso con el fin de satisfacer la demanda de agua de un sector agrícola en regadío muy desarrollado y productivo. Este sistema cuenta con 12 desaladoras, numerosos puntos de bombeos, depuradoras cuyos caudales se reutilizan en agricultura y dos trasvases, uno desde la cabecera del río Tajo (Castilla-La Mancha) y otro desde el embalse del Negratín (Andalucía Oriental).

El principal objetivo de este trabajo es valorar el impacto económico que los previsibles efectos de CC tendrán sobre la agricultura en regadío de la DHS, y evaluar las posibles medidas de adaptación para paliarlos. Para ello, se plantean tres escenarios climáticos: uno que representaría una situación actual sin $\mathrm{CC}$, y que sirve de marco de referencia, y dos de las proyecciones climáticas futuras más utilizadas en este tipo de análisis (RCP 4.5 y RCP 8.5).

\section{Metodología}

La metodología se desarrolla en la DHS, que es la unidad territorial de gestión de recursos hídricos que comprende la cuenca hidrográfica del río Segura y las cuencas costeras adyacentes. Se ubica geográficamente en el sureste de la Península Ibérica siendo su clima semiárido (Mapa 1). Dispone de escasos recursos hídricos naturales para las demandas existentes, sobre todo para su agricultura en regadío, cuyas 174.121 hectáreas (valor neto) suponen el 80\% del total del agua demandada en la DHS [CHS (2020)].

La primera fase de la metodología consiste en la evaluación de recursos hídricos en régimen natural de la DHS. Se emplea el modelo hidrológico SIMPA para evaluar el escenario de referencia sin CC, empleando para ello la serie de datos históricos entre los años 1940 y 2010 (setenta años hidrológicos consecutivos). Posteriormente, los resultados obtenidos se adaptan a las proyecciones climáticas RCP 4.5 y RCP 8.5 según la propuesta del CEDEX para el periodo 2040-2070 [Barranco et al. (2018)]. Para el escenario RCP 4.5 los caudales obtenidos se reducen un $11 \%$ y para RCP 8.5 se reducen un $23 \%$. También se ha incluido el efecto del CC para los recursos de los trasvases: aplicado los mismos valores de reducción para el caso del Negratín y los caudales del Tajo en cada escenario son los estimados por Pellicer-Martínez y Martínez Paz (2018) en un estudio específico. Un resumen de los recursos empleados viene recogido en el Cuadro 1. 
Mapa 1. Localización, zonas de riego y red hidrológica de la DHS

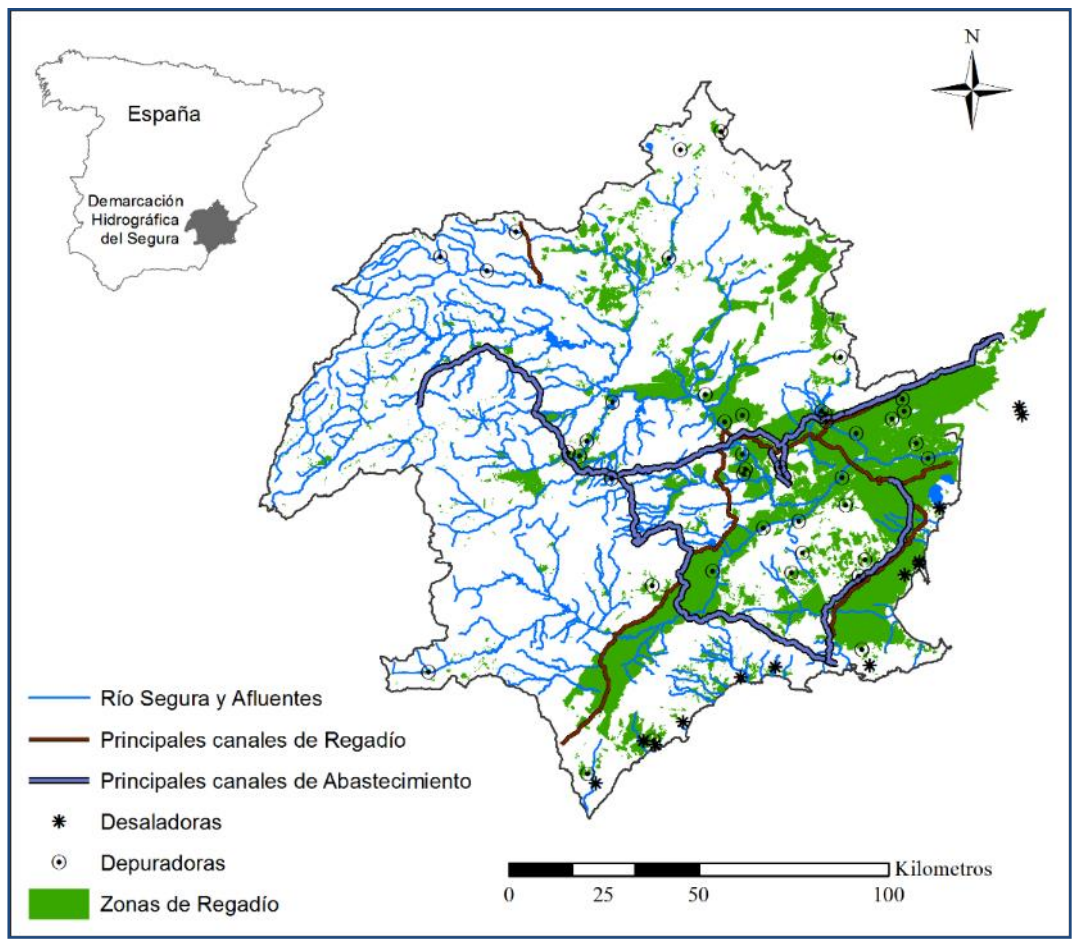

Las series de caudales obtenidas se utilizan como inputs en la segunda fase de la metodología, que es la simulación del sistema integral de recursos hídricos de la DHS, utilizando para ello el SSD-Aquatool [Pedro-Monzonís et al. (2016)]. En el diseño de este modelo de red de flujo se han considerado todas las demandas consuntivas existentes (abastecimiento, industrial y regadío) así como la oferta de los diferentes orígenes de recurso (superficial, subterráneo, reutilización, desalación y trasvases). Se ha considerado el estado actual, o de referencia (año 2018), y otro futuro dentro del periodo 2040-2070 de las proyecciones climáticas RCP (año 2050) que recoge las principales modificaciones que plantea la Confederación Hidrográfica del Segura (CHS) a futuro, como son leves aumentos de la demanda urbana y de la capacidad de desalación de la cuenca (Cuadro 1).

Cuadro 1. Caracterización de los escenarios de análisis $\left(\mathrm{Hm}^{3} / a \tilde{n} o\right)$

\begin{tabular}{|c|c|c|c|c|}
\hline & & 2018 & 2050 (RCP 4.5) & 2050 (RCP 8.5) \\
\hline \multirow{2}{*}{$\begin{array}{l}\text { Requerimientos } \\
\text { ambientales }\end{array}$} & Caudal ecológico & 0 & 0 & 0 \\
\hline & Demanda ambiental & 32 & 32 & 32 \\
\hline \multirow{3}{*}{ Demandas } & Abastecimiento y turismo* & 194 & 208 & 208 \\
\hline & Regadío* & 1.481 & 1.481 & 1.481 \\
\hline & Industrial & 20 & 20 & 20 \\
\hline \multirow{4}{*}{$\begin{array}{l}\text { Recursos } \\
\text { Hídricos }\end{array}$} & Régimen natural (incluidos subterráneos) & 1.010 & 899 & 778 \\
\hline & Desalación & $204(339)^{* *}$ & $266(339) * *$ & $305(339)^{* *}$ \\
\hline & Trasvase Tajo & 305 & 107 & 77 \\
\hline & Trasvase Negratín & 17 & 15 & 13 \\
\hline
\end{tabular}

* Valores dentro de la DHS ya que hay demandas fuera de este ámbito que se satisfacen desde la DHS

** Volumen desalado (Capacidad máxima de desalación).

La valoración económica de los déficits de suministro que se originarían por el CC se ha realizado mediante la modelización por programación lineal de los cultivos según unidades de demanda agraria agregada (UDAAs). Esta agregación de regadíos sigue los mismos criterios de zonificación climática de la propia CHS. El objetivo de la programación lineal es maximizar el margen bruto (MB) simulando el comportamiento de los agricultores ante una variación de la dotación disponible de agua [Griffin (2006)]. Las variables de decisión son las superficies de cultivo y las cantidades de agua asignadas según fuente (superficial, subterránea, regenerada y desalada) a cada UDAA. Las restricciones de dicho modelo son, básicamente, la superficie total regada, la superficie de cultivos que pueden pasar de regadío a secano, la superficie que puede pasar a barbecho, la superficie de cítricos que necesitan riegos de socorro, la superficie total de invernaderos y el agua disponible. El modelo permite la sustitución entre variedades de cultivo de regadío y secano acordes a las especializaciones productivas de cada UDAA. Todos los coeficientes técnicos del programa (rendimientos, consumos, margen bruto unitario, precios de agua según fuente de suministro) se han obtenido de la información disponible para la zona, estando los valores monetarios 
referidos al año 2018. La disponibilidad de agua para cada UDAA se ha estimado como el promedio de toda la serie simulada con SSD-Aquatool para cada escenario en términos netos, y diferenciada por fuente de suministro.

\section{Resultados}

El Cuadro 2 recoge los suministros de agua a la agricultura en regadío para cada escenario simulado. Para el escenario 2018 se suministra de media, $1.458 \mathrm{hm}^{3} /$ año, por lo que solamente habría un déficit medio del $2 \%$, pudiendo decirse que el sistema se encuentra actualmente en equilibrio (Cuadro 1). No obstante, este equilibrio se alcanza a costa de la sobreexplotación de los acuíferos, ya que la recarga natural para la DHS para el escenario 2018 se estima en unos $200 \mathrm{hm}^{3} /$ año. Para el escenario de la proyección RCP 4.5 el déficit medio de suministro se incrementa hasta el 7\%, a pesar de que se incrementa la desalación para regadío en $22 \mathrm{hm}^{3} /$ año. Por último, para la proyección RCP 8.5, el déficit de suministro alcanzaría un $11 \%$, la desalación para regadío se incrementaría, y la sobreexplotación de acuíferos incrementaría a niveles difícilmente sostenibles en el tiempo.

Cuadro 2. Suministros brutos al regadío según origen simulados para cada escenario (Hmªño)

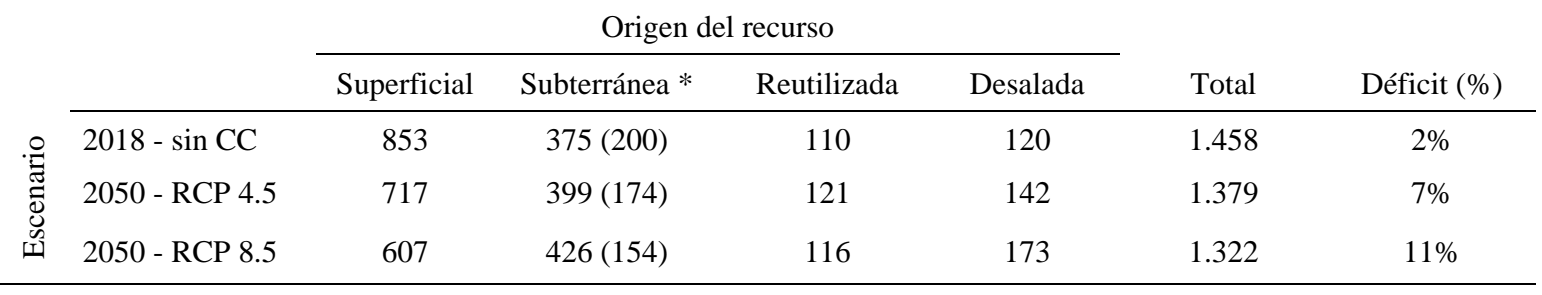

* Volumen agua subterránea utilizada (recarga natural)

Cuadro 3. Variaciones en el suministro neto, superficie de regadio y margen bruto $(M B)$ por escenario (cambios porcentuales relativos al escenario de referencia)

\begin{tabular}{lcccccc}
\hline & \multicolumn{3}{c}{ RCP 4.5 } & \multicolumn{3}{c}{ RCP 8.5 } \\
\hline & $\begin{array}{c}\text { \% Suministro } \\
\text { neto }\end{array}$ & \% Regadí & $\%$ MB & $\begin{array}{c}\text { \% Suministro } \\
\text { neto }\end{array}$ & \% Regadí & $\%$ MB \\
\hline Demarcación del Segura & $-5,3 \%$ & $-4,7 \%$ & $-4,0 \%$ & $-9,3 \%$ & $-7,4 \%$ & $-7,3 \%$ \\
UDAA 1 - Vega Alta & $-5,1 \%$ & $-5,0 \%$ & $-1,0 \%$ & $-9,7 \%$ & $-9,5 \%$ & $-2,0 \%$ \\
UDAA 2 - Vega Media/Baja & $-7,8 \%$ & $-6,1 \%$ & $-9,8 \%$ & $-12,9 \%$ & $-10,0 \%$ & $-16,4 \%$ \\
UDAA 3 - Centro Norte & $-7,5 \%$ & $-11,5 \%$ & $-1,8 \%$ & $-12,5 \%$ & $-16,7 \%$ & $-3,0 \%$ \\
UDAA 4 - Centro Sur & $-6,5 \%$ & $-7,2 \%$ & $-6,9 \%$ & $-11,1 \%$ & $-12,3 \%$ & $-11,6 \%$ \\
UDAA 5 - Litoral & $-3,3 \%$ & $0,0 \%$ & $-2,6 \%$ & $-6,9 \%$ & $0,0 \%$ & $-5,9 \%$ \\
UDAA 6 - Noroeste & $-6,2 \%$ & $-8,8 \%$ & $-1,7 \%$ & $-10,4 \%$ & $-12,1 \%$ & $-4,7 \%$ \\
UDAA 7 - Noreste & $-1,2 \%$ & $0,0 \%$ & $0,0 \%$ & $-1,7 \%$ & $0,0 \%$ & $-0,5 \%$ \\
\hline
\end{tabular}

Nota: La superficie bajo "riego de socorro" también se incluye dentro de la categoría Regadío

El Cuadro 3 muestra las variaciones porcentuales en los suministros netos, la superficie de regadío y en MB debidos a los cambios en la disponibilidad de agua para riego en cada escenario. En términos agregados para la DHS, el modelo predice una reducción de la superficie de regadío del $-4,7 \%$ en el escenario RCP 4.5 y del -7,4\% en el escenario RCP 8.5, con unas pérdidas de MB del -4,0\% y del -7,3\%, respectivamente. No obstante, los cambios a nivel de UDAA son heterogéneos y vienen explicados por las diferencias en la orientación productiva y el mix de fuentes de recursos hídricos. Los resultados muestran grandes diferencias en la reducción de la superficie de regadío que va desde el 0,0\% en la UDAA 7 Noreste, hasta el -16,7\% en la UDAA 3 - Centro Norte para el escenario de mayor reducción de recursos hídricos disponibles. En efecto, la primera de ellas sufre un menor descenso en el suministro neto ya que el agua de riego procede en su totalidad de recursos superficiales y subterráneos de coste relativamente inferior al resto de zonas, y porque se ha considerado en el modelo de gestión de agua la opción de sobreexplotar los acuíferos. En general, las UDAAs que dependen de recursos desalados y con una mayor participación de leñosos de regadío son las que sufren los mayores descensos en la superficie regada. Cabe señalar el resultado obtenido para la UDAA 5 - Litoral en la que, a pesar de que no se produciría una variación en la superficie regada, sí se produciría un aumento en la superficie de cítricos que necesitarían riegos de socorro. En particular, la 
superficie bajo este régimen de riego pasaría del 12,4\% del regadío total (escenario de referencia) hasta el $17,6 \%$ (RCP 4.5) y 23,3\% (RCP 8.5). Hay que indicar que existe la posibilidad de que parte de estas necesidades se vengan cubriendo con recursos de origen subterráneo no registrados, y que por tanto no se hayan considerado en este trabajo.

La reducción en la superficie de riego también conlleva una reducción en el MB en todas las UDAAs. En general, los mayores descensos en MB tienen lugar en aquellas zonas donde el agua desalada gana una mayor participación (UDAAs 2, 4 y 5), la cuál es creciente a medida que se reducen los recursos superficiales disponibles. No obstante, los resultados obtenidos en la UDAA 5 Litoral sugieren que una mayor especialización en cultivos de alta rentabilidad (hortícolas y hortícolas protegidos), y eficientes en el uso de agua, ayudaría a mitigar los efectos de la escasez en los escenarios analizados. Asimismo, los resultados muestran que una transformación de cultivos hacia orientaciones en secano, que sean relativamente rentables, también ayudaría a mitigar los descensos en MB en las UDAAs situadas más al interior (UDAAs 1, 6 y 7).

\section{Conclusiones}

Este trabajo valora el impacto económico del CC en la agricultura en regadío de la DHS. Para ello se ha simulado, de forma completa, el sistema de explotación de recursos hídricos junto al sistema de cultivos por unidades de demanda agraria agregadas para tres escenarios. Uno actual que toma de referencia el año 2018 y dos a futuro con las proyecciones climáticas RCP 4.5 y RCP 8.5. Los resultados sugieren una disminución de la superficie de regadío y una reducción general del Margen Bruto en todas las UDAAs, aunque de diferente intensidad en cada una. Así, se observa que las respuestas adaptativas de la agricultura de regadío en la DHS pasan por dos enfoques, reflejo de la dualidad del agro de la cuenca del Segura. Por un lado, la transformación hacia regadíos tecnificados de alta eficiencia en el uso de recurso hídrico y alta rentabilidad; por otro lado, la reintroducción de secano tradicional en aquellas zonas de climatología más favorable. Actualmente, la realidad muestra una mayor tendencia en la adopción del primer enfoque, junto a una escasa efectividad administrativa para evitar la sobreexplotación de los recursos subterráneos.

Con el fin de realizar una mejor asignación y uso de los recursos hídricos y garantizar las rentas de los agricultores, se debería evaluar la introducción de variedades de cultivo más resistentes a las sequías, el diseño de seguros de riesgos de sequía o la reasignación de derechos de agua entre los usuarios de la DHS. Así, estas medidas necesitan de una mayor coordinación entre las políticas agrarias y de agua, pero también de conservación de la naturaleza, a distintos niveles administrativos. En cualquier caso, para informar dichas políticas, serían necesarios análisis adicionales a los realizados en este trabajo, y que tengan en cuenta los impactos del CC y la escasez hídrica sobre el precio del recurso hídrico, los rendimientos de los cultivos o los mercados agrarios, entre otros.

\section{Agradecimientos}

Este trabajo se ha realizado en el marco del proyecto 20912/PI/18 financiado por la "Fundación SénecaAgencia de Ciencia y Tecnología de la Región de Murcia”

\section{Bibliografía}

CHS (2020). Plan Hidrológico de la Demarcación Hidrográfica del Segura. Revisión de tercer ciclo (20212027). Confederación Hidrográfica del Segura. Ministerio de Agricultura, Alimentación y Medio Ambiente. Murcia.

Pellicer-Martínez, F. y Martínez-Paz, J.M. (2018). "Climate change effects on the hydrology of the headwaters of the Tagus River: implications for the management of the Tagus-Segura transfer". Hydrology and Earth System Sciences, 22, 6473-6491.

Barranco, L.M., Dimas, M, Jiménez, A. y Estrada, F. (2018). "Nueva evaluación del impacto futuro del cambio climático en los recursos hídricos en España”. Ingeniería Civil, 191.

Pedro-Monzonís, M., Jiménez-Fernández, P., Solera, A., Jiménez-Gavilán, P. (2016). "The use of AQUATOOL DSS applied to the System of Environmental-Economic Accounting for Water (SEEAW)". Journal of Hydrology, 533, 1-14.

Griffin, R.C. (2006). Water resource economics: the analysis of scarcity, policies and projects. Massachusetts Institute of Technology, Cambridge. 